\title{
Anxiety and depression among Tibetan inpatients with cancer: a multicenter investigation
}

\author{
Yang Wang ${ }^{1 \#}$, Chaorong Mei ${ }^{2 \#}$, Yan Fu ${ }^{3}$, Zhiying Yue ${ }^{3}$, Yu Jiang ${ }^{4}$, Jiang Zhu $^{3}$ \\ ${ }^{1}$ Department of Oncology, 363 Hospital, Chengdu, China; ${ }^{2}$ Department of Oncology, Hospital of Chengdu Office of People's Government of \\ Tibetan Autonomous Region (Hospital.C.T), Chengdu, China; ${ }^{3}$ Department of Thoracic Oncology, West China Hospital of Sichuan University, \\ Chengdu, China; ${ }^{4}$ Department of Head and Neck Cancer, West China Hospital of Sichuan University, Chengdu, China \\ Contributions: (I) Conception and design: J Zhu, Y Jiang; (II) Administrative support: J Zhu, Y Jiang; (III) Provision of study materials or patients: Y \\ Wang, C Mei, Y Fu, Z Yue; (IV) Collection and assembly of data: Y Wang, Y Fu, Z Yue; (V) Data analysis and interpretation: Y Wang, C Mei; (VI) \\ Manuscript writing: All authors; (VII) Final approval of manuscript: All authors. \\ \#These authors contributed equally to this work. \\ Correspondence to: Jiang Zhu. Department of Thoracic Oncology, West China Hospital of Sichuan University, 37 Guoxue Lane, Chengdu, China. \\ Email: hxnadal@163.com.
}

Background The Tibetan population is minority in southwest China, and data on the psychological states
of Tibetan cancer inpatients are not available. The study participants included Tibetan and Han cancer
inpatients, and their depression and anxiety were investigated and analyzed to understand the psychological
states of Tibetan cancer patients. The aim of the present study was to understand the incidence of depression
and anxiety among Tibetan cancer inpatients, and the factors affecting their depression and anxiety. Methods: We used questionnaires to investigate the anxiety and depression of Tibetan and Han cancer inpatients. The questionnaires included the Zung Self-Rating Anxiety Scale, Zung Self-Rating Depression Scale, and a general information questionnaire.

Results: The results showed that there were 61 cases (53\%) of depression and 43 (37.4\%) of anxiety among Tibetan cancer inpatients, and 27 cases (23.5\%) and $16(13.9 \%)$ among Han cancer patients. The major factors affecting depression among Tibetan cancer patients were permanent address and type and stage of cancer; the major factors affecting anxiety among Tibetan cancer patients were education level and type and stage of cancer.

Conclusions: The incidence of depression and anxiety among Tibetan cancer inpatients was significantly greater than that of Han cancer inpatients. The major factors affecting the incidence of depression and anxiety were permanent address, education level, and type and stage of cancer. We suggest that further research should be directed at mental health problems among Tibetan cancer patients in order to determine the best possible psychological interventions.

Keywords: Tibetan; cancer; depression; anxiety; questionnaire

Submitted Jul 29, 2020. Accepted for publication Sep 23, 2020.

doi: 10.21037/apm-20-1721

View this article at: http://dx.doi.org/10.21037/apm-20-1721

\footnotetext{
$\wedge$ ORCID-0000-0001-6386-6117.
} 


\section{Introduction}

Cancer is a chronic, non-infectious disease that has seriously effects on physical and mental health; its incidence increases annually in China. In 2015, there were approximately 4,290,000 new cancer patients in China (1), and in 2016, cancer was the most dominant cause of death. The World Health Organization (WHO) states that cancer is a sociopsychological disease (2), and that cancer patients will present negative emotional responses to diagnosis and treatment, them often experience multiple distressing symptoms simultaneously; depression and anxiety are the most common emotional responses. There are numerous reports on the correlation between concomitant depression and anxiety in cancer patients worldwide. According to Yang et al., the incidence of depression is $54.9 \%$ and the incidence of anxiety is $49.69 \%$ for domestic cancer patients, which are significantly higher than the incidence of depression (17.5\%) and the incidence of anxiety (18.37\%) for the general population (3). Studies have shown that the incidence of depression and anxiety globally among cancer patients is $3-58 \%$ and $1.3-23 \%$, respectively $(4,5)$. China is a multinational country, and Tibetan cancer patients mainly live in Tibet, Qinghai, Sichuan, and Yunnan. However, data on depression and anxiety among Tibetan cancer inpatients are not available. In the present study, the Chinese Zung Self-Rating Depression Scale (SDS) and Zung Self-Rating Anxiety Scale (SAS) (6) were translated into Tibetan to evaluate the suitability of the Tibetan versions. The aim of the present study was to explore the occurrence of depression and anxiety among Tibetan cancer inpatients and the correlation between various factors (e.g., type of cancer, sex, age, marital status, permanent address, education level, career, religious beliefs, and stage of cancer) influencing depression and anxiety to provide a basis for subsequent correlational studies on psychological interventions.

We present the following article in accordance with the SURGE reporting checklist (available at http://dx.doi. org/10.21037/apm-20-1721).

\section{Methods}

\section{General materials}

The present study was approved by the ethics committees of the involved hospitals (No.: 2018-11), and informed consent was obtained from all of the patients. All procedures performed in this study involving human participants were in accordance with the Declaration of Helsinki (as revised in 2013). The patients were recruited through posters directed at Tibetan and Han cancer patients admitted to the Department of Oncology, Hospital of Chengdu Office of People's Government of Tibetan Autonomous Region (Hospital.C.T); Department of Oncology, 363 Hospital; and Cancer Center, West China Hospital of Sichuan University from January 2016 to January 2018. The total number of Tibetan and Han patients recruited was 245. The inclusion criteria were as follows: (I) no mental health issues before cancer diagnosis by cytological or histological pathological examination; (II) could clearly understand and complete the questionnaires; and (III) at least 18 years old. The exclusion criteria were as follows: (I) concomitant mental symptoms and affective mental disorders, or use of psychotropic drugs before cancer diagnosis; (II) serious cognitive disorder and alogia; and (III) $<18$ years old. Patients who did not complete the questionnaires or providing conflicting responses to the questions were also excluded.

\section{Study methods}

\section{Suitability of test scales: Tibetan version}

The Chinese SDS and SAS were translated into Tibetan by Tibetan members from the Tibetan Medicine Office of 363 Hospital, and then back-translated by specialists proficient in Chinese and Tibetan for cross-cultural availability. A presurvey involving 20 Tibetan patients who could effectively communicate in both Tibetan and Chinese was conducted to analyze the reliability and validity of the Tibetan versions of the SDS and SAS, and appropriate amendments were made.

\section{Questionnaire}

The questionnaire survey with unified instruction was applied by investigators specially trained before the formal survey. The selected patients independently completed the questionnaires within 30 minutes. All completed questionnaires were kept by the document controller at the Department of Oncology, 363 Hospital.

\section{Survey tools}

A background questionnaire was created. The question included age, sex, marital status, permanent address, education level, career, and religious beliefs. The Tibetan version of the SDS was used to evaluate depression among Tibetan cancer inpatients in the past week. The standard score threshold was set to 53 (in the Chinese normal model), the standard mild depression scores ranged from 
53 to 62 , the moderate depression scores ranged from 63 to 72 , and the severe depression score was $\geq 73$ (7). The Tibetan version of the SAS was used to evaluate anxiety among Tibetan cancer inpatients in the past week. The standard score threshold was set to 50 (in the Chinese normal model), the standard mild anxiety scores ranged from 50 to 59 , the moderate anxiety scores ranged from 60 to 69 , and the severe anxiety score was $\geq 70$ (7). For the Han cancer inpatients, we used the Chinese versions of the SDS and SAS to assess symptom severity. The standard score threshold was set to that of the Tibetan version.

\section{Statistical analysis}

We used EpiData 3.1 software for the double data input of questionnaires, and analyzed these using SPSS version 21.0. We presented continuous variable data as mean value \pm standard deviation. Student's $t$-test was used to compare two groups, and analysis of variance was used to compare multiple groups. The rank sum test was used to analyze ranked data, and $\chi^{2}$-test was used for classified variables. Binary logistic regression analysis was used to analyze the factors influencing depression and anxiety among patients. The level of significance was $\alpha=0.05$, and the difference was statistically significant at $\mathrm{P}<0.05$.

\section{Results}

\section{Study completion and baseline characteristics}

A total of 245 questionnaires were distributed, of which 238 were completed (93.9\%). Of these, eight were considered invalid due to being incomplete. The Tibetan cancer patients were aged $23-80$ years, with an average age of $56.03 \pm 11.6$ years. There were 74 male patients $(64 \%)$ and 41 female patients (36\%); 59 patients were from the Tibet Autonomous Region, 55 were from Sichuan Province, and 1 was from another province. There were 16 patients with respiratory cancer, 65 with digestive system neoplasms, 6 with reproductive cancers, 9 with head and neck neoplasms, and 19 with other cancers. Forty-five patients were illiterate, 30 had a primary school education, 21 had a junior high school education, 3 had a senior high school education, and 16 had a college degree or higher.

The Han cancer patients were aged $27-87$ years old, with an average age of $55.04 \pm 11.8$ years. There were 75 male patients (65\%) and 40 female patients (35\%); 2 were from the Tibet Autonomous Region, 103 were from Sichuan
Province, and 10 were from other provinces. There were 20 patients with respiratory cancers, 68 with digestive system neoplasms, 2 with reproductive cancers, 7 with head and neck neoplasms, and 18 with other cancers. Four patients were illiterate, 21 had a primary school education, 34 had a junior high school education, 24 had a senior high school education, and 32 had a college degree or higher. The differences between these two groups of cancer patients were statistically significant, except for, age, marital status, and type of cancer.

\section{Incidence of depression}

The incidence of depression among the Tibetan cancer inpatients was $53 \%$. There were 61 cases of depression among the Tibetan cancer inpatients, including 29 with mild depression (25.2\%), 27 with moderate depression (23.5\%), and 5 with severe depression $(4.3 \%)$. The incidence of depression among the Han cancer inpatients was $23.5 \%$. There were 27 cases of depression among the Han cancer inpatients, including 13 with mild depression (11.3\%), 9 with moderate depression (7.8\%), and 5 with severe depression (4.3\%). The test revealed that the incidence of depression among Tibetan cancer inpatients was significantly higher than that of the Han cancer inpatients, and the difference was statistically significant $(\mathrm{P}<0.0001)$ (Table 1).

\section{Incidence of anxiety}

The incidence of anxiety among the Tibetan cancer inpatients was $37.4 \%$. There were 43 cases of anxiety among the Tibetan cancer inpatients, including 21 with mild anxiety (18.3\%), 18 with moderate anxiety (15.7\%), and 4 with severe anxiety (3.5\%). The incidence of anxiety among the Han cancer inpatients was $13.9 \%$. There were 16 cases of anxiety among the Han cancer inpatients, including 8 with mild anxiety (7\%), 5 with moderate anxiety (4.3\%), and 3 with severe anxiety (2.6\%). The test revealed that the incidence of anxiety among the Tibetan cancer inpatients was significantly higher than that of the Han cancer inpatients, and the difference was statistically significant $(\mathrm{P}<0.0001)$ (Table 2).

\section{Multifactor analysis}

\section{Factors influencing depression among the Tibetan cancer patients}

The multifactor analysis of the 115 Tibetan cancer patients 
Table 1 Incidence of depression: Tibetan $v s$. Han patients

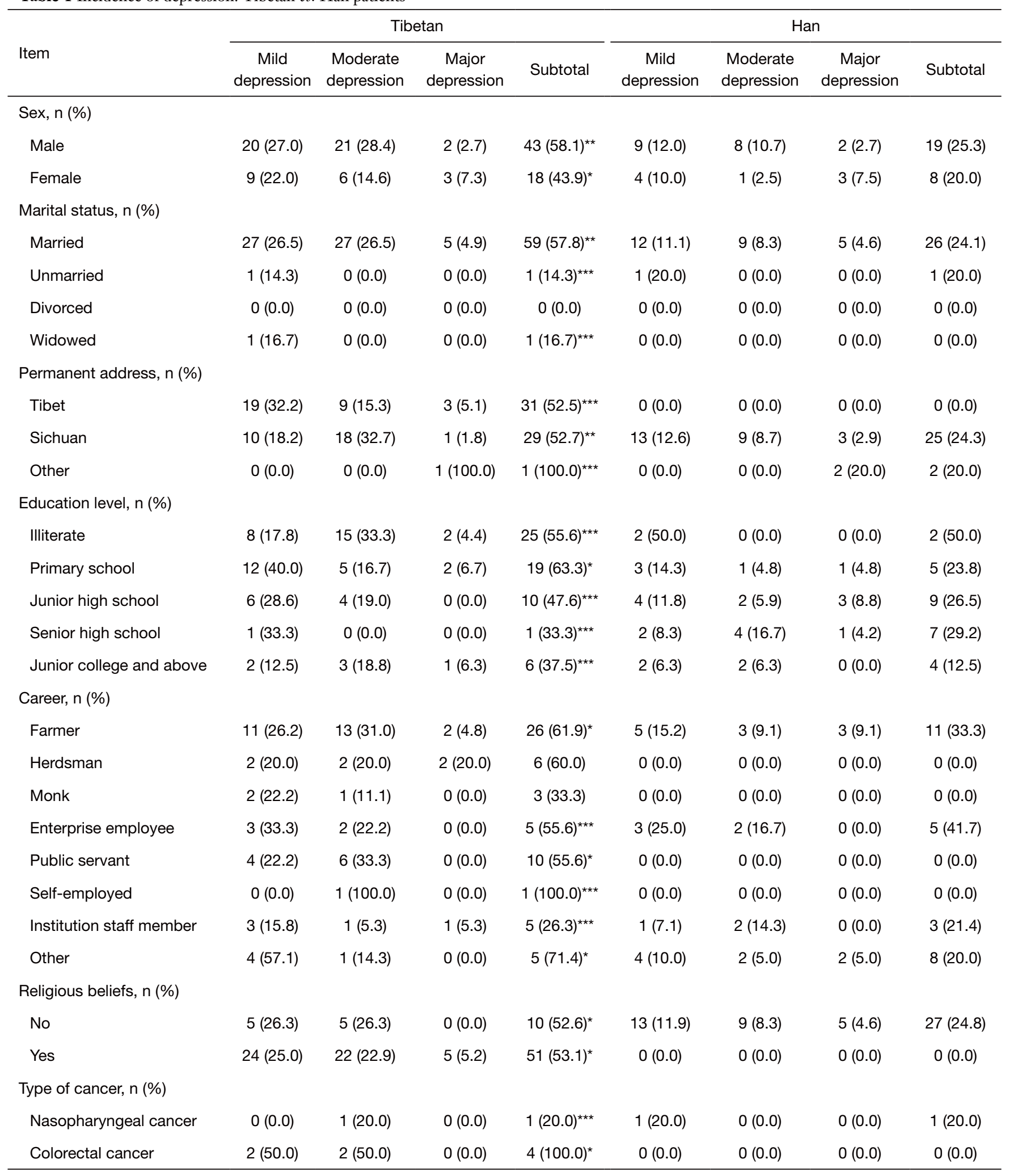

Table 1 (continued) 
Table 1 (continued)

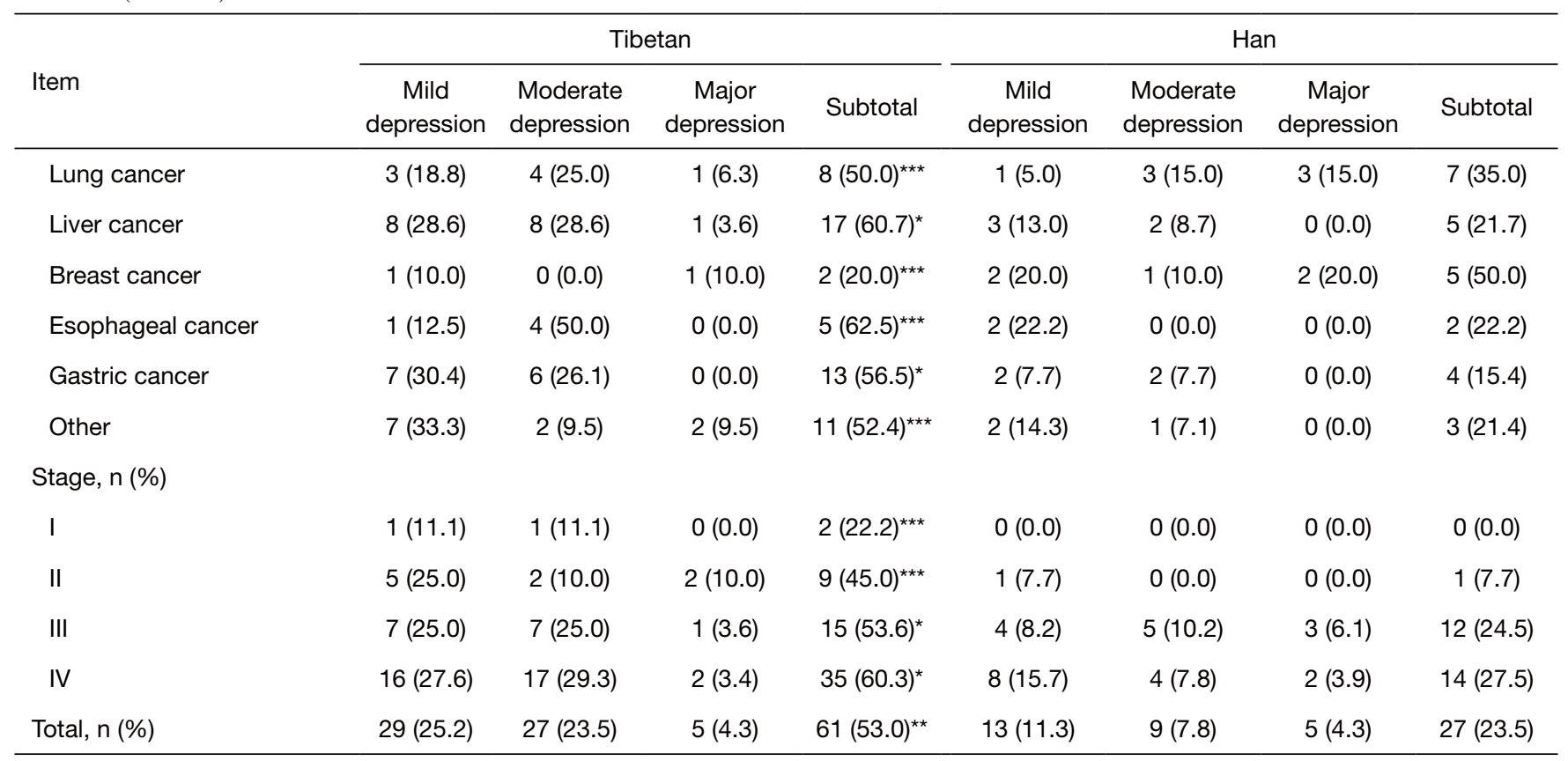

${ }^{\star}, \mathrm{P}<0.05 ;{ }^{* \star}, \mathrm{P}<0.0001 ;{ }^{* \star \star}, \mathrm{P}>0.05$.

Table 2 Incidence of anxiety: Tibetan patients $v s$. Han patients

\begin{tabular}{|c|c|c|c|c|c|c|c|c|}
\hline Item & \multicolumn{4}{|c|}{ Tibetan } & \multicolumn{4}{|c|}{ Han } \\
\hline \multicolumn{9}{|l|}{ Sex, n (\%) } \\
\hline Male & $14(18.9)$ & $10(13.5)$ & $3(4.1)$ & $27(36.5)^{\star}$ & $7(9.3)$ & $3(4.0)$ & $2(2.7)$ & $12(16.0)$ \\
\hline \multicolumn{9}{|c|}{ Marital status, n (\%) } \\
\hline Married & $19(18.6)$ & $17(16.7)$ & $4(3.9)$ & $40(39.2)^{\star \star}$ & $7(6.5)$ & $5(4.6)$ & $3(2.8)$ & $15(13.9)$ \\
\hline Unmarried & $1(14.3)$ & $0(0.0)$ & $0(0.0)$ & $1(14.3)^{\star \star \star}$ & $1(20.0)$ & $0(0.0)$ & $0(0.0)$ & $1(20.0)$ \\
\hline Divorced & $0(0.0)$ & $0(0.0)$ & $0(0.0)$ & $0(0.0)$ & $0(0.0)$ & $0(0.0)$ & $0(0.0)$ & $0(0.0)$ \\
\hline \multicolumn{9}{|c|}{ Permanent address, n (\%) } \\
\hline Sichuan & $11(20.0)$ & $9(16.4)$ & $2(3.6)$ & $22(40.0)^{\star \star}$ & $7(6.8)$ & $5(4.9)$ & $2(1.9)$ & $14(13.6)$ \\
\hline Other & $0(0.0)$ & $1(100.0)$ & $0(0.0)$ & $1(100.0)^{\star \star *}$ & $1(10.0)$ & $0(0.0)$ & $1(10.0)$ & $2(20.0)$ \\
\hline \multicolumn{9}{|c|}{ Education level, n (\%) } \\
\hline Illiterate & $10(22.2)$ & $9(20.0)$ & $0(0.0)$ & $19(42.2)^{\star \star \star}$ & $0(0.0)$ & $0(0.0)$ & $0(0.0)$ & $0(0.0)$ \\
\hline Primary school & $3(10.0)$ & $6(20.0)$ & $3(10.0)$ & $12(40.0)^{\star \star}$ & $1(4.8)$ & $1(4.8)$ & $1(4.8)$ & $3(14.3)$ \\
\hline
\end{tabular}

Table 2 (continued) 
Table 2 (continued)

\begin{tabular}{|c|c|c|c|c|c|c|c|c|}
\hline Item & \multicolumn{4}{|c|}{ Tibetan } & \multicolumn{4}{|c|}{ Han } \\
\hline Senior high school & $1(33.3)$ & $0(0.0)$ & $0(0.0)$ & $1(33.3)^{\star \star \star}$ & $1(4.2)$ & $1(4.2)$ & $0(0.0)$ & $2(8.3)$ \\
\hline Farmer & $10(23.8)$ & $9(21.4)$ & $2(4.8)$ & $21(50.0)^{\star \star}$ & $3(9.1)$ & $0(0.0)$ & $0(0.0)$ & $3(9.1)$ \\
\hline Herdsman & $0(0.0)$ & $1(10.0)$ & $1(10.0)$ & $2(20.0)$ & $0(0.0)$ & $0(0.0)$ & $0(0.0)$ & $0(0.0)$ \\
\hline Monk & $1(11.1)$ & $1(11.1)$ & $0(0.0)$ & 2 (22.2) & $0(0.0)$ & $0(0.0)$ & $0(0.0)$ & $0(0.0)$ \\
\hline Institution staff member & $2(10.5)$ & $2(10.5)$ & $1(5.3)$ & $5(26.3)^{\star \star \star}$ & $0(0.0)$ & $0(0.0)$ & $0(0.0)$ & $0(0.0)$ \\
\hline Other & $1(14.3)$ & $2(28.6)$ & $0(0.0)$ & $3(42.9)^{\star \star \star}$ & $3(7.5)$ & $3(7.5)$ & $3(7.5)$ & $9(22.5)$ \\
\hline \multicolumn{9}{|l|}{ Religious beliefs, n (\%) } \\
\hline No & $6(31.6)$ & $2(10.5)$ & $0(0.0)$ & $8(42.1)^{\star}$ & $8(7.3)$ & $5(4.6)$ & $3(2.8)$ & $16(14.7)$ \\
\hline Yes & $15(15.6)$ & $16(16.7)$ & $4(4.2)$ & $35(36.5)^{\star \star \star}$ & $0(0.0)$ & $0(0.0)$ & $0(0.0)$ & $0(0.0)$ \\
\hline \multicolumn{9}{|l|}{ Type of cancer, n (\%) } \\
\hline Nasopharyngeal cancer & $0(0.0)$ & $0(0.0)$ & $0(0.0)$ & $0(0.0)$ & $0(0.0)$ & $0(0.0)$ & $0(0.0)$ & $0(0.0)$ \\
\hline Gastric cancer & $5(21.7)$ & $3(13.0)$ & $0(0.0)$ & $8(34.8)^{\star}$ & $2(7.7)$ & $0(0.0)$ & $0(0.0)$ & $2(7.7)$ \\
\hline Other & $3(14.3)$ & $3(14.3)$ & $2(9.5)$ & $8(38.1)^{\star \star \star}$ & $1(7.1)$ & $0(0.0)$ & $0(0.0)$ & $1(7.1)$ \\
\hline \multicolumn{9}{|l|}{ Stage, n (\%) } \\
\hline I & $1(11.1)$ & $0(0.0)$ & $0(0.0)$ & $1(11.1)^{\star \star \star}$ & $0(0.0)$ & $0(0.0)$ & $0(0.0)$ & $0(0.0)$ \\
\hline II & $2(10.0)$ & $0(0.0)$ & $2(10.0)$ & $4(20.0)^{\star \star \star}$ & $0(0.0)$ & $0(0.0)$ & $0(0.0)$ & $0(0.0)$ \\
\hline III & $6(21.4)$ & $4(14.3)$ & $0(0.0)$ & $10(35.7)^{\star}$ & $2(4.1)$ & $2(4.1)$ & $1(2.0)$ & 5 (10.2) \\
\hline IV & $12(20.7)$ & $14(24.1)$ & $2(3.4)$ & $28(48.3)^{\star}$ & $6(11.8)$ & $3(5.9)$ & $2(3.9)$ & $11(21.6)$ \\
\hline Total, n (\%) & 21 (18.3) & $18(15.7)$ & $4(3.5)$ & $43(37.4)^{\star \star}$ & $8(7.0)$ & $5(4.3)$ & $3(2.6)$ & 16 (13.9) \\
\hline
\end{tabular}

*, $\mathrm{P}<0.05 ;{ }^{* *}, \mathrm{P}<0.0001 ;{ }^{* * \star}, \mathrm{P}>0.05$ 
Table 3 Binary logistic regression analysis of factors influencing depression among Tibetan cancer patients

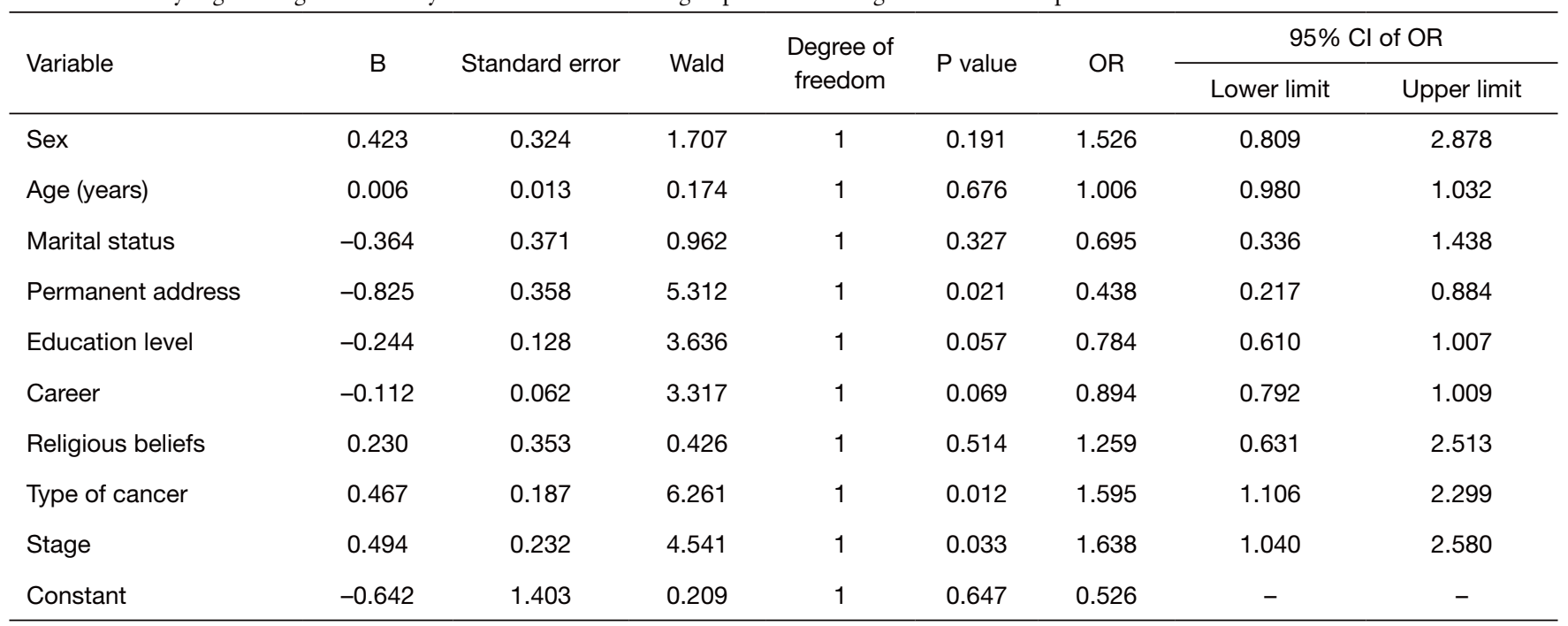

$\mathrm{Cl}$, confidence interval; OR, odds ratio.

showed that the factors resulting in a high incidence of depression among these patients were permanent address [odds ratio (OR): $0.438,95 \%$ confidence interval (CI): $0.217-0.884$ ], type of cancer (OR: 1.595 , 95\% CI: $1.106-$ 2.299), and cancer stage (OR: 1.638, 95\% CI: 1.040-2.580). There were no statistically significant differences for sex, age, marital status, education level, career, and religious beliefs (Table 3).

\section{Factors influencing anxiety among the Tibetan cancer patients}

The factors resulting in a high incidence of anxiety among the Tibetan cancer patients were education level (OR: 0.746, 95\% CI: 0.562-0.991), type of cancer (OR: 2.067, 95\% CI: 1.325-3.223), and cancer stage (OR: 1.959, 95\% CI: 1.164 3.295). There were no statistically significant differences for sex, age, marital status, permanent address, career, and religious beliefs (Table 4).

\section{Discussion}

Cancer is a common and frequently occurring disease that has serious impacts on physical and mental health. The Tibetan population is a minority in southwest China, and data on the incidence of depression and anxiety among Tibetan cancer inpatients are not available. In the present study, we showed that the Tibetan cancer inpatients had relatively severe psychological problems, with a significantly higher incidence of depression and anxiety than that the
Han cancer inpatients $(\mathrm{P}<0.0001)$.

Tibetan cancer patients receiving offsite medical treatment often have challenges due to communication and differences in culture and religious beliefs. This increases the incidence of depression and anxiety, in combination with the adverse effects of the cancer.

The binary logistic regression analysis showed that the regional environment, type and stage of cancer had an impact on depression among Tibetan cancer inpatients, and education level and type and stage of cancer had an impact on anxiety. According to Peng et al., although the incidence of depression and anxiety as different among patients with different cancers, further comparisons showed no statistical significance (8).

\section{Study limitations}

Pain, reduced household income, medical insurance payment modes, and other factors that impact the mental well-being of cancer patients were not considered during the study design. Studies in China have shown that the average scores for depression and anxiety in patients with no pain are significantly lower than those for patients with pain, and the number of patients with depression among those with no pain is significantly less than those with pain. The duration and maximum intensity of pain had a significantly positive correlation with depression, and pain relief had a significantly negative correlation (9). It is necessary to include additional factors influencing depression and 
Table 4 Binary logistic regression analysis of factors influencing anxiety among Tibetan cancer patients

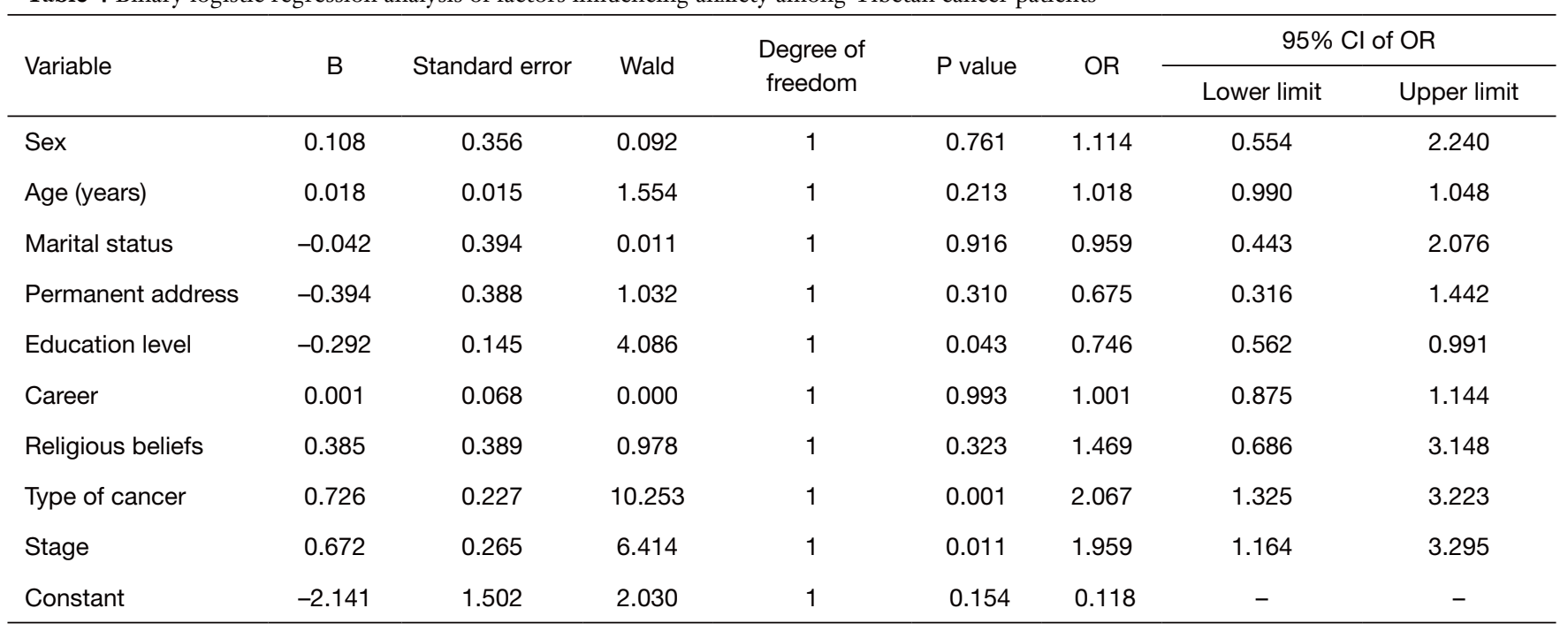

$\mathrm{Cl}$, confidence interval; OR, odds ratio.

anxiety in these questionnaires in future studies, and to conduct further studies in the local hospitals of the Tibetan population for complete and reliable results. The present study used a small sample size, and a representative sample size was further reduced after stratification; therefore, the efficiency of the statistical test was reduced. The sample size of future studies should be increased, and the statistical analysis should be improved to provide a scientific basis for late psychological intervention.

\section{Conclusions}

Based on the findings of the present study, we recommend that medical personnel consider the mental health problems of minority patients with cancer to improve the early recognition rate and the treatment of cancer patients with depression and anxiety in order to minimize their length of hospital stay, medical expenses, and psychological distress, and to improve their treatment compliance and quality of life. It is important to further explore the mental health of Tibetan and other Chinese minorities with cancer to promote the balanced development of all nationalities, maintain social security, and unity and promote social harmony and stability (10).

\section{Acknowledgments}

Funding: None.

\section{Footnote}

Reporting Checklist: The authors have completed the SURGE reporting checklist. Available at http://dx.doi. org/10.21037/apm-20-1721

Data Sharing Statement: Available at http://dx.doi. org/10.21037/apm-20-1721

Conflicts of Interest: All authors have completed the ICMJE uniform disclosure form (available at http://dx.doi. org/10.21037/apm-20-1721). The authors have no conflicts of interest to declare.

Ethical Statement: The authors are accountable for all aspects of the work in ensuring that questions related to the accuracy or integrity of any part of the work are appropriately investigated and resolved. All procedures performed in this study involving human participants were in accordance with the Declaration of Helsinki (as revised in 2013). The present study was approved by the ethics committees of the involved hospitals (No.: 2018-11), and informed consent was obtained from all of the patients.

Open Access Statement: This is an Open Access article distributed in accordance with the Creative Commons Attribution-NonCommercial-NoDerivs 4.0 International License (CC BY-NC-ND 4.0), which permits the noncommercial replication and distribution of the article with 
the strict proviso that no changes or edits are made and the original work is properly cited (including links to both the formal publication through the relevant DOI and the license). See: https://creativecommons.org/licenses/by-nc-nd/4.0/.

\section{References}

1. Chen W, He J. CA: Cancer statistics in China, 2015. Medical Innovation of China 2016;5:149.

2. Li X. Cancer and depressive disorders. Clinical Journal of Chinese Medicine 2012;4:1-4.

3. Yang YL, Liu L, Wang Y, et al. The prevalence of depression and anxiety among Chinese adults with cancer: a systematic review and meta-analysis. BMC Cancer 2013;13:393.

4. Deimling GT, Wagner LJ, Bowman KF, et al. Coping among older-adult, long-term cancer survivors.

Psychooncology 2006;15:143-59.

Cite this article as: Wang Y, Mei C, Fu Y, Yue Z, Jiang Y, Zhu J. Anxiety and depression among Tibetan inpatients with cancer: a multicenter investigation. Ann Palliat Med 2020;9(6):3776-3784. doi: 10.21037/apm-20-1721
5. Brown LF, Kroenke K, Theobald DE, et al. The association of depression and anxiety with health-related quality of life in cancer patients with depression and/or pain. Psychooncology 2010;19:734-41.

6. Wang X, Wang X. Rating Scales for Mental Health. Beijing: Chinese Mental Health Journal 1999:235-8.

7. Li X, Yue W. Medical Psychology. Edition 2. Beijing: People's Military Medical Press 2009:958.

8. Peng J, Zhou L, Zhou H, et al. Investigation and analysis on depression and anxiety emotion of 100 cases of tumors. Chongqing Medicine 2015;44:1949-53.

9. Li X, Xiao W, Jiao S, et al. A retrospective cohort study on anxiety and depression in cancer pain patients. Chinese Journal of Pain Medicine 2016;22:354-9.

10. Zhang L, Yang G, Li C, et al. Advance in Study on Mental Health of Minority Groups in China. Journal of Regional Anatomy and Operative Surgery 2015;24:450-3. 